\title{
Development of On-board Weighting and Force Sensor System for Construction Machines: Static Analysis.
}

\author{
Fiorenzo Malaguti \\ CEMOTER-C.N.R., via Canal Bianco 28, 44044 Cassana(FE), Italy \\ fax +39-532-732250 E-mail:malaguti@cemoter.bo.cnr.it
}

\begin{abstract}
This paper considers an on-board system for construction machines to measure weight of handling or excavated materials and/or contact force of machine tool or end effector for construction robot.

Weight and force are measured indirectly by the differential pressure of hydraulic actuators of machines, driving the machine arms.

This system improves the commercial weighting system, because it uses the load pressures of two actuators to detect every time the exact load position.

At the same time it is able to measure direction and value of contact force without changes of hardware, moreover, it may be used with a large types of machines.

This system is developed for construction robot, but it could be use for present commercial machines as weighting system and warning system to detect buried obstacles.
\end{abstract}

\section{Introduction}

Automation and robotics for construction machines to work in its operating environment need sensor comparatively not expensive and reliable.

The use of the differential pressure of hydraulic actuator, driving the bucket or forklift, to measure weight or contact forces is a simple and not expensive method, used for the present commercial on-board weighting system.

Really the differential or load pressure doesn't measure a force but the load torque, the load force is carried out supposing known the load application point. The present weighting systems use many contrivances to fix the load application point and to improve measure precision, these methods or contrivances aren't yet compatible with the development of sensors suitable for autonomous or automated machines, therefore, to improve performance and measure the load for every arm and tool positions, a second load pressure and a continuous displacement sensor for an other actuator, were introduced, so as a tilt sensors to detect arm attitute respect to inertial reference.

The system was developed from beginning as contact force sensor for automated construction machines, but being usable as weighting system, this characteristic is here shown with emphasis.

The system is able to carry out by displacement, pressure and tilt measurements the value and application point or direction of load forces, at same time it's possible to transform it ever by software in a weighting system

The relationship to use the system as weighting and contact forces sensor are shown, these relationships are based about a static analysis of forces and torques , acting on the tool or end effector and arms or links of machines.

A small analysis of error is made too, to know the contributions to final error of measures errors.

The system is made by pressure and displacement transducers and a CPU, to compute data, but it uses machine hardware as the mechanical part of itself.

Depending the system on machine design and configuration a short analysis on machine kinematics is shown.

\section{Kinematics of machines}

Usually arms and booms of construction machines are driven by hydraulic cylinders, their linear displacements and forces are converted in angular displacements and torques by crank mechanisms or links.

Supposing these links perfectly stiff, the angular displacement of the tool and other links is related to linear displacement of cylinder rod.

The measurement system considers the mechanisms and cylinder driving the tool and the mechanism and cylinder driving the arm or link on which there is the rotation pin of tool. 
Usually this last one is a simple sliding crank mechanism (fig. 1), where the $\theta$ angle is related to the cylinder extension by the following relationship,

$\theta=\arccos \left(\frac{L_{2}^{2}+f^{2}-r^{2}}{2 L_{2} f}\right)$

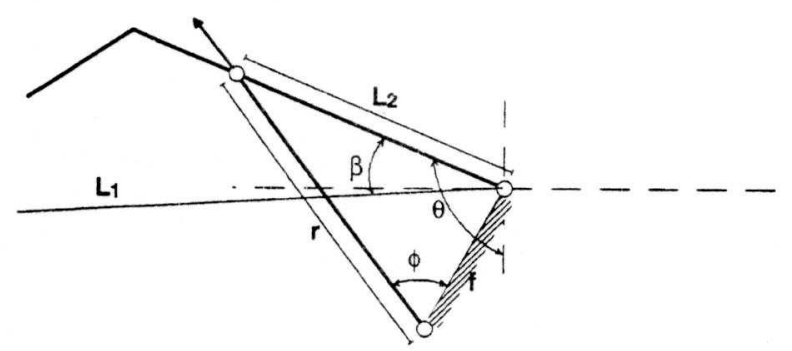

fig. 1

while the produced torque is related to load force of cylinder by

$T_{2}=F_{2} L_{2} \sin (\theta+\phi)$

with $\phi$ function of $\theta$.

$\phi=\arctan \frac{L_{2} \sin \theta}{f-L_{2} \cos \theta}$

The mechanisms driving the tool are more complex and have several configurations, so the angular positions of tool and the torque on it shall be related to extension of relative cylinder in general by trigonometric relationships.

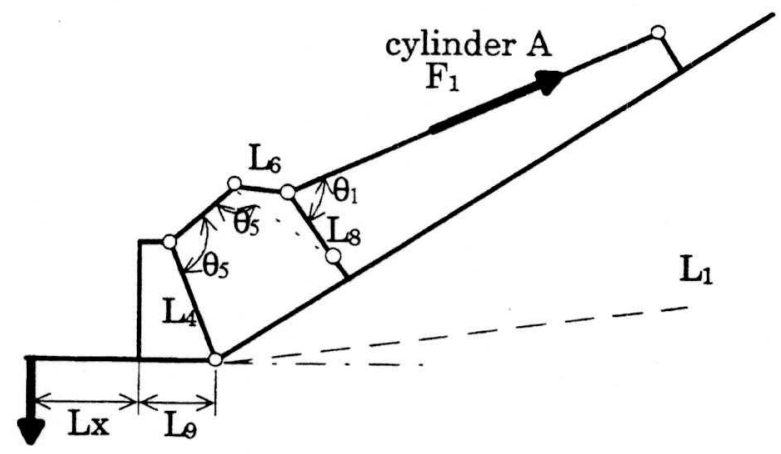

fig. 2

For example, the forklift shown in fig.2 [1] the trigonometric relationship, which will be named $K\left(\theta_{a f}\right)$ in the next part of work, is.

$$
\frac{L_{4} L_{8} \sin \theta_{6} \sin \theta_{1}}{\left(L_{x}+L_{9}\right) L_{6} \sin \theta_{5}}
$$

\section{Static analysis of force}

As shown above, a system, based on the load pressure measurement of a cylinder only, in general isn't accurate because the application point of load isn't known exactly.

To know this point, in implicit or explicit way, it must have a second relationship, to solve two equations system with two unknowns.

The system considers two static equilibrium equations of forces, respect to load and the differential pressures of cylinders driving the tool and the last arm.

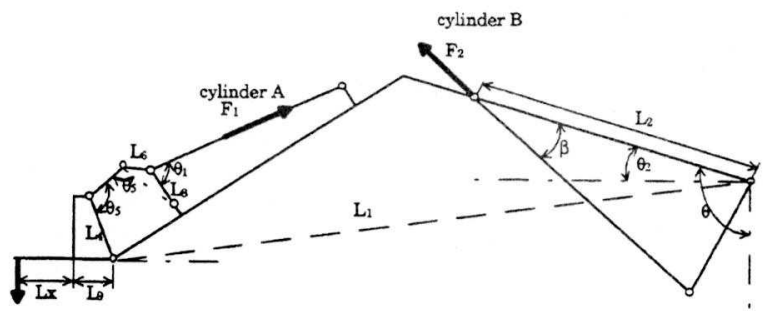

fig. 3

Let consider as example the mechanisms shown in figure 3 , which is like an arm of loader equipped with forklift.

Putting the static equilibrium between load and the forces provided by cylinders, it obtains two relationships (3), where $K_{T}\left(\theta_{a f}\right)$ is the transmission ratio between the first cylinder force and load torque, $\beta$ is the angle between $L_{1}$ and $L_{2}, \theta_{a f}$ is the angle between the arm and tool, $\theta_{x}$ is the angle between tool and horizontal axes, $h_{l}$ and $h_{2}$ are the tares.

$$
\begin{aligned}
& W\left[\left(L_{x}+L_{9}\right) \cos \theta_{x}+L_{1} \cos \left(\beta-\theta_{2}\right)\right]= \\
& F_{2} L_{2} \sin (\theta+\phi)-h_{2}\left(\theta_{a f}, \phi\right) \\
& W\left(L_{x}+L_{9}\right) \cos \theta_{x}=F_{1} K_{T}\left(\theta_{a f}\right)-h_{1}\left(\theta_{a f}, \theta\right)
\end{aligned}
$$

The angle $\theta_{2}$ and $\phi$ are function of $\theta$, therefore, it's easy to show the relationship of load to have the following form.

$W=f\left(\theta_{a f}, \theta\right) F_{2}+g\left(\theta_{a f}, \theta\right) F_{1}-h\left(\theta_{a f}, \theta\right)$ 
This relationship shows the possibility to carry out the load value by the measures of load pressures of two cylinder and the angular positions of tool and arm (or the rod positions of cylinder).

As the $\theta$ angle needs an absolute reference, the system needs a tilt sensor to have the vertical axes or the machines attitude.

\section{Static analysis of force as contact sensor}

Previously the static analysis for the weighting system has been shown, now it shows as the system may become a contact force sensor.

An automatic machine as a excavator robot needs a contact force sensor to detect obstacles and planing trajectories. Since isn't easy to detect the exact contact point when the tool is into the soil and some times it may be useful to known the direction of contact force in addition to its value, a sensor based on a cylinder is inadequate.

The proposed sensor allows to detect the value of contact force and the contact point or the value and direction of this force.

As the first case is similar to weighting system, here the second case shall be consider, for this aim a simple kinematic solution for excavator has been used (fig.4).

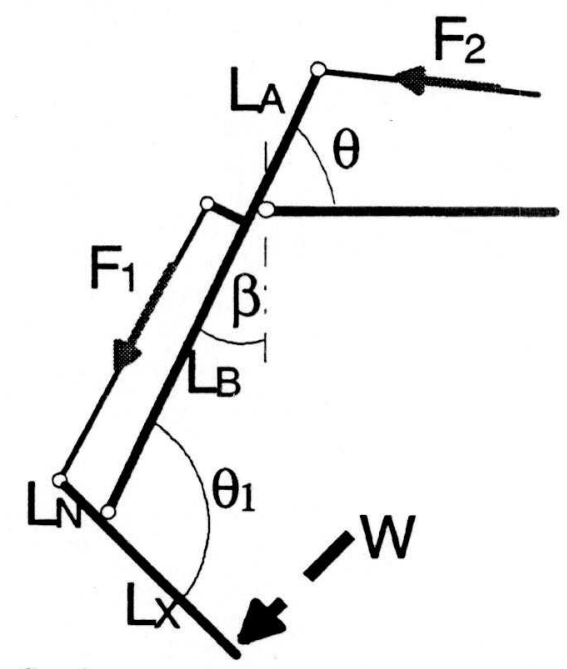

fig. 4

The two equations of equilibrium are,

$$
\begin{aligned}
& W L_{x} \sin \alpha=F_{1} L_{n} \sin \left[\Phi_{1}\left(\pi-\theta_{1}\right)\right]-h_{1}\left(\theta_{1}, \theta\right) \\
& W\left[L_{x} \cos \left(\pi-\theta_{1}\right)+L_{B}\right] \cos \left[\alpha-\left(\frac{\pi}{2}-\theta_{1}\right)\right]= \\
& =F_{2} L_{A} \sin \Phi(\theta)-h_{2}\left(\theta_{1}, \theta\right)
\end{aligned}
$$

where $\Phi_{I}$ and $\Phi$ are two angles depending respectively on $\theta_{l}$ and $\theta$, while $\alpha$ is the angle between the direction of contact force $W$ and $L_{x}$.

Solving these equations shall be possible to carry out $W$ and $\alpha$.

\section{Measurement errors}

To identify quite a force on the plane, where the arms, boom and tool move, it needs three variables, while the system to measure forces or weight proposed here is able to carry out two variables only.

The reasons of this limitation are the following:

- the more simple machines as loader haven't other arms or link with hydraulic cylinder to measure an other load pressure;

- the costs or better the performance-cost ratio

- in fact, other position and pressure transducers increase the cost, but introduce errors.

As seen above the force measured by the load pressure of hydraulic cylinders is the sum of load and tare. The tare is due to the mass of arm driven by the cylinder and the mass (or better the moments of inertia) of above arms and tool, so a modest percentage error of measure may introduce large error on the measure of load.

A small analysis of error propagation is here considered to verify the influence of many parameters on measurement accuracy.

Given the generic relationship of measurement

$g=f\left(u_{1}, u_{2}, \ldots, u_{n}\right)$

it's possible to compute the final error $\Delta g$ expanding $g$ by Taylor's series and using the first order term only.

$$
\Delta g=\left|\Delta u_{1} \frac{\partial f}{\partial u_{1}}\right|+\left|\Delta u_{2} \frac{\partial f}{\partial u_{2}}\right|+\cdots+\left|\Delta u_{n} \frac{\partial f}{\partial u_{n}}\right|
$$
$W$,

Expanding by Tailor's series the relationship (4) of

$$
\begin{aligned}
& \Delta W=\left|\Delta \theta \frac{\partial f\left(\theta_{a f}, \theta\right)}{\partial \theta} F_{2}\right|+\left|\Delta \theta_{a f} \frac{\partial g\left(\theta_{a f}, \theta\right)}{\partial \theta_{a f}} F_{1}\right|+ \\
& \left|f\left(\theta_{a f}, \theta\right) \Delta F_{2}\right|+\left|g\left(\theta_{a f}, \theta\right) \Delta F\right|_{1}+ \\
& \left|\frac{\partial h_{1}\left(\theta_{a f}, \theta\right)}{\partial \theta_{a f}} \Delta \theta_{a f}\right|+\left|\frac{\partial h_{2}\left(\theta_{a f}, \theta\right)}{\partial \theta} \Delta \theta\right|
\end{aligned}
$$


it may note four error terms depending on kinematic measurement (angles $\theta$ and $\theta_{a f}$ ), that can compensate one another in part, while errors of pressure measures depend on total error directly.

As one can see, the force $F_{2}$ and its errors of measurement could influence the final error heavily, because they have higher values respect $F_{l}$. A third pair of position and force measurements up the previous arm could introduce other and larger errors.

\section{Improvements}

The availability of horizontal position respect to a fix reference allows to carry out additional informations, one of that can be the position of the center of load mass on the working plane of arm and tool.

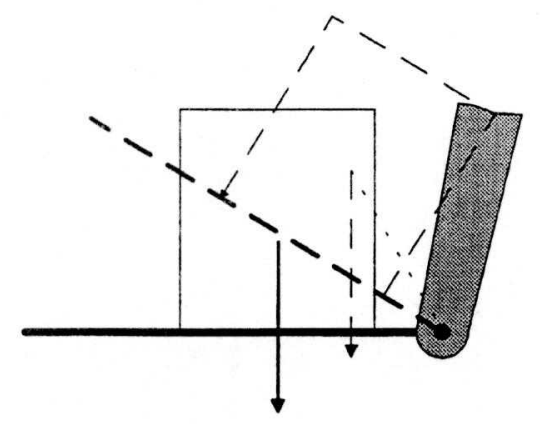

fig. 5

Rotating the tool only from its horizontal position, knowing the horizontal position of weight and the angle of rotation, one may compute the height of centre of mass respect to the tool.

The same result may be obtained, beginning from a generic position, knowing the initial angular position of tool respect to horizontal.

Fitting up the arm by an hydraulic "wrist", a force or its component placed on a parallel plane to arm one and perpendicular to arm axes may be measured.

\section{Conclusion}

A force and torque sensor with many degree of freedom designed just for construction machines, could be too expansive to have a wide diffusion, one thinks, in fact, the expensive and complex system could inhibit the automation and robotics in construction machinery field

In this work is presented a system, based on the hardware and plants of machine, which allows to measure, or better to compute, the value and a second characteristic of a force on the operating plane of boom, arms and tool of machines.
This apparent limitation is counterbalanced by the use of not expensive transducers of pressure and displacement, non high performance CPU, a present technology.

The system, used as weighting system, has a wide area of employment, because it may equip construction machines, so as agricultural, industrial and service machine, moreover, it may be used too as warning sensor during soil excavation, for example detecting a limit of soil cutting effort to not damage buried pipe, without changes of system hardware.

For a robot in continuous contact with environment a force sensor is indispensable, moreover, the use of measures of torque driving the joint to estimate the load is enchanting, some new research topics about robot control involve just on regressive methods to estimate the load characteristics from driving torques and displacements of joints.

The direct hydraulic actuators allow to obtain easily these measures, in particular by the type of our joint control used for excavator robot [5], these are in practice already available.

To weight a load or measure a force in movement the difficulties increase, because the measures must be made in dynamic conditions, they are affected moreover by disturbs introduced by the movement of all part of machine, main frame, wheels, tracks included.

We hope in a next future to solve these problems to have an effective force sensor for automated construction machines.

\section{References}

[1] Otani R., Nishizaki K., Shibata Y., Susawa K., Tamaki K., Weighting system using tractor-mounted frond-end loader, Conference AIGR, Milan,1994

[2] Sirohi R.S., Radha Krishna H.C., Mechanical Measurement, Wiley Eastern Limited, 1980

[3] Paul B., Kinematics and dynamics of planar machinery, Prentice-Hall Inc., Englewood Cliffs, 1979

[4] Huang X. D., Bernold L. E., Robotic rock handling during backhoe excavation, $10^{\text {th }}$ ISARC, Houston, USA, 24-26 May, 1993

[5] Malaguti F., Variable Structure Control in Excavator Robot, 11th ISARC, Brighton, U.K., 24-26 May, 1994 